The helium legacy of dead stars

Mon. Not. R. Astron. Soc. (in the press);

Preprint astro-ph/0302285 at <http://arXiv.org > (2003)

Findings from studies of the first stars in

the Universe may upset standard models

of the Big Bang. According to R. Salvaterra

and A. Ferrara, these objects, called

Population III stars, might have produced

an appreciable fraction of the helium-4

$\left({ }^{4} \mathrm{He}\right)$ found in interstellar space and thought previously to have been created mainly in the Big Bang itself.

Already, models of element formation in the Big Bang don't quite match up with observations of the amounts of these 'primordial' elements in the Universe: there seems to be rather less ${ }^{4} \mathrm{He}$ and ${ }^{7} \mathrm{Li}$ than expected. If much of this ${ }^{4} \mathrm{He}$ was made by fusion of hydrogen in Population III stars, that puts the predictions even further astray.

When subatomic particles first condensed from the Big Bang, they formed mostly ${ }^{1} \mathrm{H}$. About $10 \%$ of this matter was ${ }^{4} \mathrm{He}$, with smaller amounts of ${ }^{2} \mathrm{H},{ }^{3} \mathrm{He}$ and ${ }^{7} \mathrm{Li}$. But ${ }^{4} \mathrm{He}$ would also have been made from ${ }^{1} \mathrm{H}$ in the first stars. The trouble is, these stars are now dead, so their abundance must be estimated indirectly. Salvaterra and Ferrara estimate it from the near-infrared background (NIRB) radiation, the glow emitted by cosmic gas warmed by starlight ever since the first stars shone. The NIRB has been measured recently, and it implies that Population III stars were more productive than previously thought.

Philip Ball

\section{Plant cell biology}

\section{Ancient rings}

Plant Cell 15, 655-665 (2003)

Mitochondria are energy-generating organelles found in all organisms; chloroplasts are the site of photosynthesis in plants. Both are thought to have originated long ago when simple, bacterium-like cells were engulfed by more complex cells. During cell proliferation these organelles must be replicated and separated, and a protein with bacterial origins, FtsZ, is involved in chloroplast division. Mitochondria in higher organisms lack FtsZ and instead use the protein dynamin, implying that chloroplasts kept the ancient division system while mitochondria gained a new one.

But Shin-ya Miyagishima et al. now report that in the primitive red alga Cyanidioschyzon merolae, chloroplasts use dynamin as well. One type of dynamin, CmDnm1, was already known to be involved in mitochondrial division in C. merolae. Through genome searches, Miyagishima et al. uncovered a related
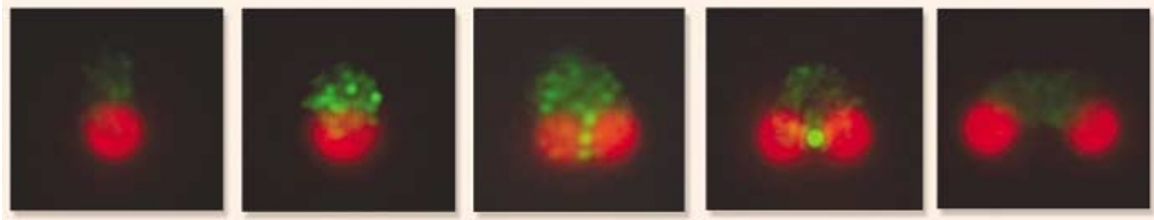

Dynamin dynamics: a chloroplast (red) is severed by a ring of dynamin protein (green).

dynamin, CmDnm2, which during cell division migrates to the chloroplast to form a ring that constricts around the chloroplast's 'waist' (as pictured here).

A relative of FtsZ had already been found to participate in mitochondrial division in C. merolae. So in this primitive plant, dynamin and FtsZ help both chloroplasts and mitochondria divide. This hints that the ancestors of these organelles likewise used rings of both proteins.

Christopher Surridge

\section{Ecology}

\section{Nuclear reptiles}

\section{Mol. Ecol. 12, 743-752 (2003)}

Lizards can be faithful too - D. O'Connor and R. Shine have discovered that the Australian black rock skink, Egernia saxatilis, forms long-term pairs to raise its offspring. The authors found from genetic tests that more than $80 \%$ of skink groups include one female adult and one male adult, with at least some male-female pairs and offspring remaining together throughout the three-year duration of the research project. Families share a territory, but spend most of their time apart.

Egernia is the most sociable lizard known -15 of the 29 species in this genus live in some form of family group. But these tend to be larger and more diffuse than those of E. saxatilis, which is the first reptile found to have a 'nuclear family' structure of a pair and offspring living together for more than one year. Fidelity in this species is not perfect, however, in that a few groups contained more than two adults, and a fifth of clutches had offspring fathered by an external male.

John Whitfield

\section{Surface chemistry}

\section{Riddle of the stones}

J. Am. Chem. Soc. 125, 2854-2855 (2003)

Kidney stones, which cause excruciating pain when they lodge in the urinary tract, are a mystery. They are typically formed by the crystallization and build-up of calcium oxalate on the surfaces of the kidney, but it isn't clear how the crystals become attached to renal cells. Xiaoxia Sheng and colleagues have gone fishing for clues at the molecular scale, using an atomic force microscope (AFM).

There is evidence that carboxylate groups in biological macromolecules might be the hooks that snag the growing crystals to cells of the renal epithelium. To test this notion, the researchers coated the tip of an AFM with long-chain organic molecules carrying a carboxylate (or other organic groups for comparison) at the free end, and waved it over the surface of single calcium-oxalate crystals.

They found that the adhesion force is several times larger for carboxylates than for other end groups, such as methyl or alcohols. And adhesion is disrupted when poly(aspartic acid) is added to the solution, suggesting that this polymer sticks to the crystal surface and exhausts all the binding sites. That squares with the fact that carboxylate-rich macromolecules — similar to poly(aspartic acid) — in urine help suppress crystal attachment to renal cells.

Philip Ball

\section{Developmental biology}

\section{Tools for single-cell analysis}

Dev. Cell 4, 383-393 (2003)

During development, immature cells are often committed to becoming specific mature cell types long before any physical signs of this commitment can be seen. Changes in gene-expression patterns are presumably the key, but most research has analysed populations of cells and so has been unable to identify any differences between individual cells.

Ming-Ko Chiang and Douglas A. Melton have now used a modified form of the polymerase chain reaction, together with DNA microarrays, to study the genes expressed by single pancreas cells from mouse embryos. The microarrays are custom-made to light up when active genes from a cell form a match with any of the known pancreas genes on the microarray chip. Chiang and Melton identified six subtypes of developing pancreas cells, each expressing a unique combination of genes, and in some subtypes they discovered combinations of four genes - $P d s 1$ to $P d s 4$ — that were not previously known to be expressed in the pancreas.

The researchers propose a model of pancreas development in which the early pancreas contains a heterogeneous mix of cells, with genetically distinct cell types becoming apparent over time. They also suggest that this type of analysis could help to characterize cell subtypes in other biological systems.

Helen R. Pilcher 